\title{
Simultaneous Ground-Based Remote Sensing of Water Vapor by Differential Absorption and Raman Lidars
}

\author{
D. D. Turner \\ Pacific Northwest National Laboratory \\ Richland, Washington \\ H. Linné, J. Bösenberg, S. Lehmann, and K. Ertel \\ Max-Planck Institute for Meteorology \\ Hamburg, Germany \\ J. E. M. Goldsmith and T. P. Tooman \\ Sandia National Laboratories \\ Livermore, California
}

\section{Introduction}

Water vapor is well known to be a critical component in many aspects of atmospheric research, such as radiative transfer and cloud and aerosol processes. The distribution of water vapor in the atmosphere is highly variable, both in time and in space, and has proven to be very difficult to measure accurately. The U.S. Department of Energy's Atmospheric Radiation Measurement (ARM) Program's overall goal is to improve global climate models by improving the treatment of the radiative transfer processes that occur in the atmosphere. One of the conclusions of the ARM Program was that uncertainties in the absolute calibration of the water vapor measurements are currently the limiting factor in the improvement of radiative transfer algorithms for clear skies (Clough et al. 1999). While instruments such as the microwave radiometer (MWR) can provide accurate measurements of total precipitable water vapor (PWV) in the column, accurate profiles of water vapor are also needed in order to calculate accurate cooling rate profiles.

Raman lidar and differential absorption lidar (DIAL) are presently the most advanced techniques to measure the vertical distribution of water vapor in the atmosphere with both high temporal and vertical resolution and accuracy. Therefore, it is important to perform a dedicated experiment to assess the performance of both systems, with respect to accuracy, resolution, and available range. A secondary goal of this experiment was to better characterize the operational Raman lidar at the ARM Southern Great Plains (SGP) Cloud and Radiation Testbed (CART) site in north central Oklahoma. The water vapor DIAL from the Max-Planck-Institute (MPI) for Meteorology in Hamburg, Germany, was collocated with the Raman lidar from September 29 to October 19, 1999. The two systems were located about $15 \mathrm{~m}$ apart, and more than $100 \mathrm{~h}$ of coincident data was collected during both daytime and nighttime. Radiosondes, which were launched from the CART site every three hours during this experiment, and collocated MWR data are also used in this study. 


\section{Instruments}

The ARM Raman lidar was designed and built for autonomous measurements of water vapor profiles at the CART site. The laser transmitter is a flashlamp-pumped Nd:YAG, with radiation from the third harmonic (at $355 \mathrm{~nm}$ ) being transmitted to the atmosphere. It uses a dual field-of-view (FOV) and narrowband interference filters to reject a large fraction of the solar background, and thus is able to profile water vapor and aerosols throughout the diurnal cycle. Automated algorithms have been developed to retrieve calibrated profiles of water vapor and aerosols, and since 1998, over $7500 \mathrm{~h}$ of data have been collected and processed. Additional details about this system are given in Goldsmith et al. (1998), and an extensive analysis of the water vapor data from this system with a wide variety of other water vapor sensors was reported in Turner and Goldsmith (1999). This eye-safe instrument was operated in its nominal mode for continuous monitoring during this experiment.

The MPI water vapor DIAL system utilizes a flashlamp-pumped Alexandrite ring laser that is injection seeded by a cw Ti:Sapphire laser. This results in narrow linewidth, high-frequency stability, and a high spectral purity of the outgoing laser beam, all of which are critical to make accurate differential absorption measurements. The system is fully described in Wulfmeyer and Bösenberg (1998). An important addition to this system that extends the maximum height to which profiles can be retrieved is the standard operation of a variable attenuator (Lehmann et al. 1997). Since this system is not eye-safe, Federal Aviation Administration (FAA) restrictions limited typical operation from local noon to midnight.

Raman lidars have been used for many years to accurately profile water vapor at night. Daytime measurements of water vapor are much more difficult due to the large increase in the solar background, and hence daytime Raman lidar measurements of water vapor are not as well characterized as nighttime measurements. The CART Raman lidar utilizes two different modes in order to profile water vapor throughout the diurnal cycle. The daytime mode differs from the nighttime mode only by the inclusion of an additional neutral density filter in the narrow FOV channel, which ensures that the detected signal is in (or near) the linear range of the detector. However, this results in approximately $90 \%$ of the total signal being thrown away during the day, and could result in a significant difference in the nighttime versus daytime abilities of this system. Since the DIAL technique looks at the elastic backscatter at two different wavelengths, its signal strengths are much greater than those observed by the Raman lidar, and thus it is less sensitive to diurnal changes in the solar background. Therefore, one goal of this study is to assess the performance of the two systems in terms of system availability, height range covered, temporal and spatial resolution, and relative absolute accuracy for both the nighttime and daytime measurements.

\section{Results}

From September 29 to October 19, the Raman lidar was operational 94\% of the time, with the exceptions being periods of normal maintenance, such as cleaning the window. During the nighttime, this system is able to profile water vapor to near the tropopause or cloud base, whichever is lower. During the daytime, though, the reduced signal-to-noise ratio results in water vapor profiles extending only to about $3 \mathrm{~km}$, depending on the averaging performed and atmospheric conditions. Since the signal 
measured is molecular Raman scattering, the returning signal is proportional to the amount of water vapor in the atmosphere, and hence daytime profiles may be restricted to lower heights in drier conditions. The nominal vertical resolution employed in the Raman lidar processing for 10-min data for both day and night is shown in Figure 1.

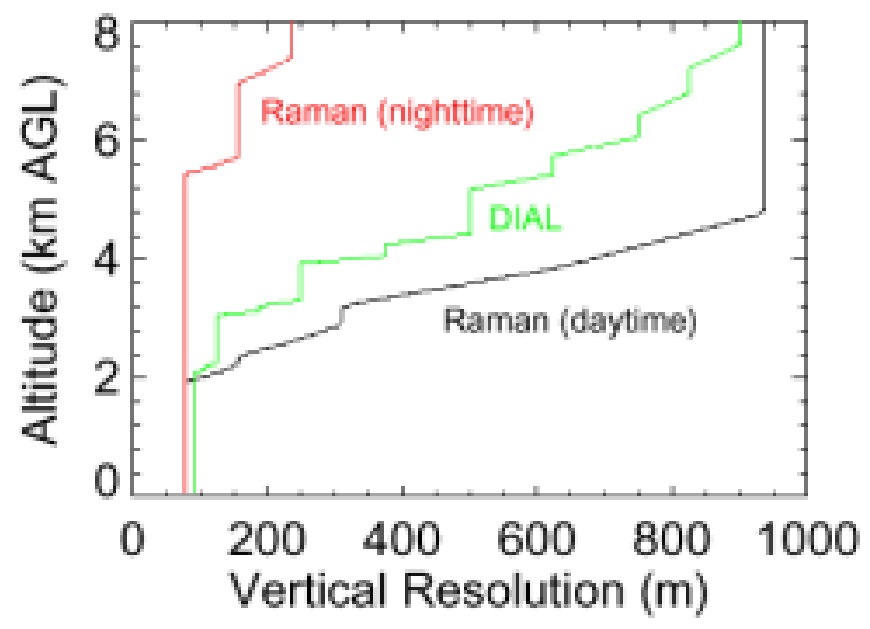

Figure 1. Vertical resolution used in this analysis.

The DIAL was designed as an experimental rather than operational system, and thus its total availability will be less than the Raman. However, of the approximately 140 hours of operation that were requested from the FAA, the system was operational nearly $75 \%$ of the time, including time periods needed for system testing. Post-analysis indicated that approximately 30 hours of this data had to be discarded because the laser transmitter was not working properly. Of the remaining 75 hours, approximately 30 hours from 4 different days (October 4, 7, 9, and 13) with different meteorological conditions have been analyzed for this paper.

While the Raman lidar's signal is proportional to the amount of water vapor in the atmosphere, large amounts of water vapor in the boundary layer restrict the maximum height range the DIAL can profile by extinguishing the on-line signal. Therefore, under favorable conditions with relatively little water vapor in the lower atmosphere, this DIAL can profile to nearly $8 \mathrm{~km}$. During this experiment, the maximum range achieved was between 6 to $8 \mathrm{~km}$. The vertical resolution of the DIAL's profiles is also shown in Figure 1. For the four days analyzed in this paper, the total PWV ranged from $1.7 \mathrm{~cm}$ to $3.0 \mathrm{~cm}$.

One way to evaluate the possible day-to-night differences in the Raman lidar is to compare water vapor profiles from the two systems as a function of solar background. Figure 2 shows three different comparisons taken on October 9 from near solar noon (left), at sunset (middle - note that the Raman lidar is still in daytime mode), and during the evening (right). These are 10-min averages from both lidars. The nearest radiosonde to these profiles has been plotted also. The "error" plots are the standard way each system reports relative error, and so the error has been computed in different manners for each system. For the Raman lidar, the error profiles are calculated using Poisson statistics from the 

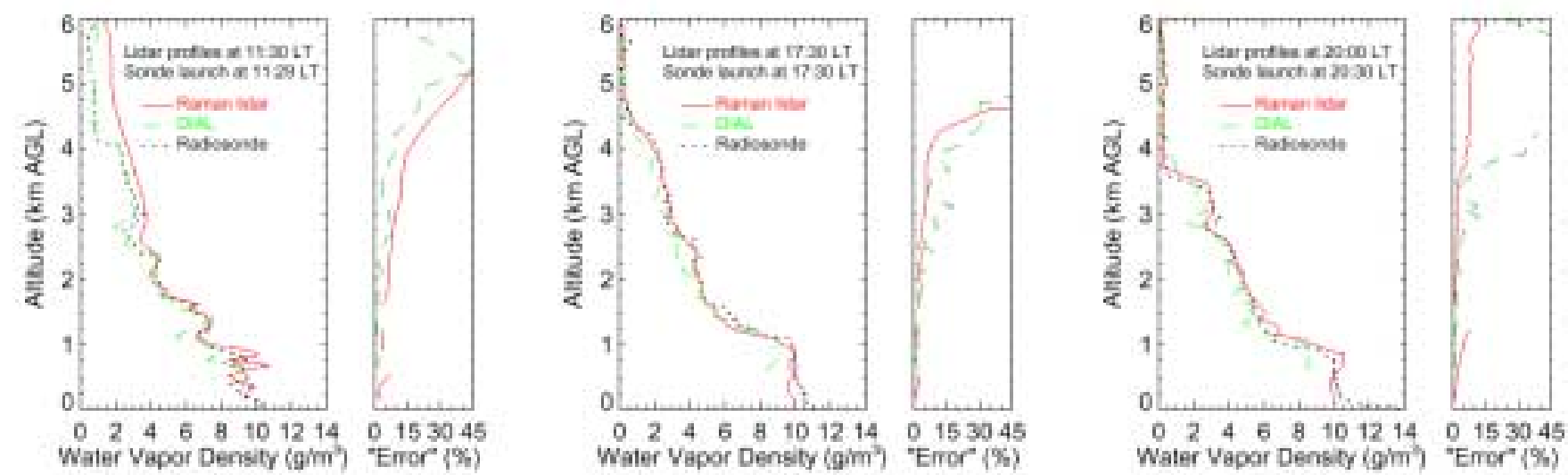

Figure 2. Water vapor profiles from October 9 from the Raman lidar, DIAL, and radiosonde near local noon (left), sunset (middle), and at night (right). The relative error profiles are computed differently for each lidar; see text for details.

backscattered counts. The DIAL error profiles are calculated from the power spectrum of the water vapor time series at each altitude, where the noise level in the spectrum is used to estimate the standard deviation in the humidity retrieval (Senff et al. 1994).

These profiles highlight the typical ability of these systems. During the daytime, the two systems agree fairly well between $1 \mathrm{~km}$ and $3 \mathrm{~km}$, above which the Raman retrievals have poorer vertical resolution and less accuracy due to the difficulty in detecting the weak Raman signals at these altitudes in the high solar background conditions. The DIAL and radiosonde, though, agree quite well up to $6 \mathrm{~km}$, although there appears to be a slight dry bias in DIAL data compared to the radiosonde. As the solar background decreases, though, the accuracy and resolution of the Raman lidar retrievals increases markedly, and is in very good agreement with the radiosonde. There also appears to be a small height offset in the DIAL data in these plots that is still under investigation. It should be noted that the 11:30 (LT) sonde is $4 \%$ drier than the MWR, the 17:30 sonde is $2 \%$ drier than the MWR, and the 20:30 sounding is in nearperfect agreement with the MWR.

Linné et al. (2000) present a more detailed investigation of these random errors, concluding that both systems have the sensitivity (random errors less than 5\%) to be used in boundary layer process studies during the night. However, only the DIAL has the required sensitivity for these studies during the day, as its random error in the boundary layer remains approximately $5 \%$, while the Raman lidar's error is approximately $15 \%$.

Because one of the ARM Program's goals is to improve radiative transfer algorithms, absolute accuracy of the water vapor measurements is a high requirement. Clough et al. (1999) have argued that the 22-GHz water vapor line is very well known, and thus the MWR can be used to measure PWV with a high absolute accuracy. The DIAL data can also be used as an absolute reference with an accuracy that is determined by the precision to which the absorption cross section of the water vapor line is known. Because the DIAL's moisture measurements did not extend to the surface, while the lowest range gate from the Raman lidar is $60 \mathrm{~m}$, we utilized the Raman lidar as a transfer standard. 
Although some new ways to absolutely calibrate Raman lidars have recently been investigated (Sherlock et al. 1999; Whiteman et al. 2000), the Raman lidar's height-independent calibration factor is determined from the MWR (Turner and Goldsmith 1999). To calibrate the Raman lidar, the first step is to merge together the profiles from the two FOVs. Since there is an extra neutral density filter in the narrow FOV during the daytime, the nighttime and daytime data are merged separately. The region in which the two FOVs are merged changes from day to night, as the merge range is a compromise between signal-to-noise in the wide FOV channel and the desire to merge as high in the atmosphere as possible to reduce the impact of any errors in the overlap correction applied to the narrow FOV data. The merged nighttime data is then integrated and compared to the MWR to derive a single heightindependent calibration factor that is applied for the entire day. This calibration process is entirely automated. However, because the Raman lidar is calibrated to the MWR daily, the Raman lidar can be used to compare the absolute accuracy of the MWR with the DIAL.

To compare the two lidar systems, we computed the integrated precipitable water vapor (IPW) content from 10-min profiles from both systems between $1 \mathrm{~km}$ and $3 \mathrm{~km}$, a region where both lidars operate well during both the day and night. The normalized difference of the two, defined as 100(IPW Raman $_{\text {- }}$

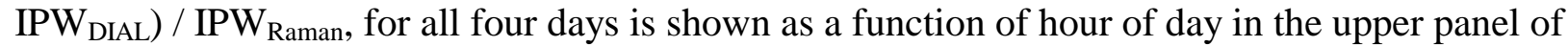
Figure 3. The transition from daytime to nighttime is defined as the time when the Raman lidar
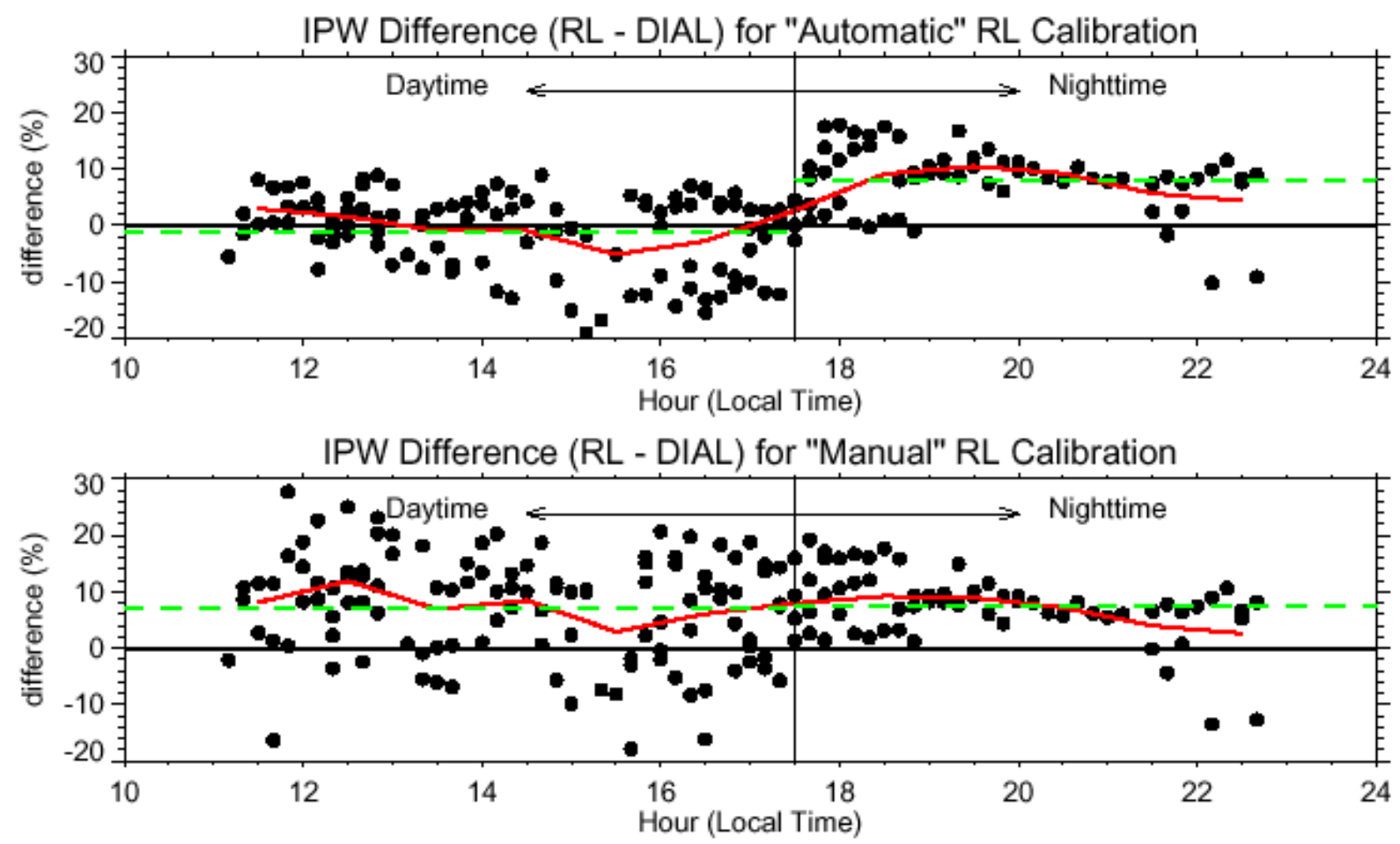

Figure 3. The normalized difference of the IPW from $1 \mathrm{~km}$ to $3 \mathrm{~km}$ from the Raman lidar and DIAL, for the original automated calibration results (top) and results where the relationship between the narrow and wide FOVs is manually determined (bottom). The solid red line indicates hourly averages of the differences, whereas the dashed green lines indicate mean values for each period. See text for details. 
transitioned to the nighttime mode, which corresponds to a solar zenith angle near 90 degrees. The red line in the figure corresponds to 1 hourly averages of the points, while the green line indicates the mean value for each of the two periods.

During the daytime, the agreement between the two lidars is within $1 \%$ to $4 \%$ with a large variation in the difference. However, during the nighttime, the difference between the two lidars is approximately $10 \%$, although the variance decreases markedly.

In order to ascertain that this day/night difference could be attributed to the Raman lidar, a similar analysis was done by comparing the Raman lidar's PWV with that derived from the MWR. Recall that the only the nighttime MWR data are used to determine a single factor used to calibrate the Raman lidar for the entire day. The normalized differences are shown in the upper panel of Figure 4. These results indicate that the change seen in the IPW differences between the two lidars is due, at least to a large extent, to the Raman lidar.

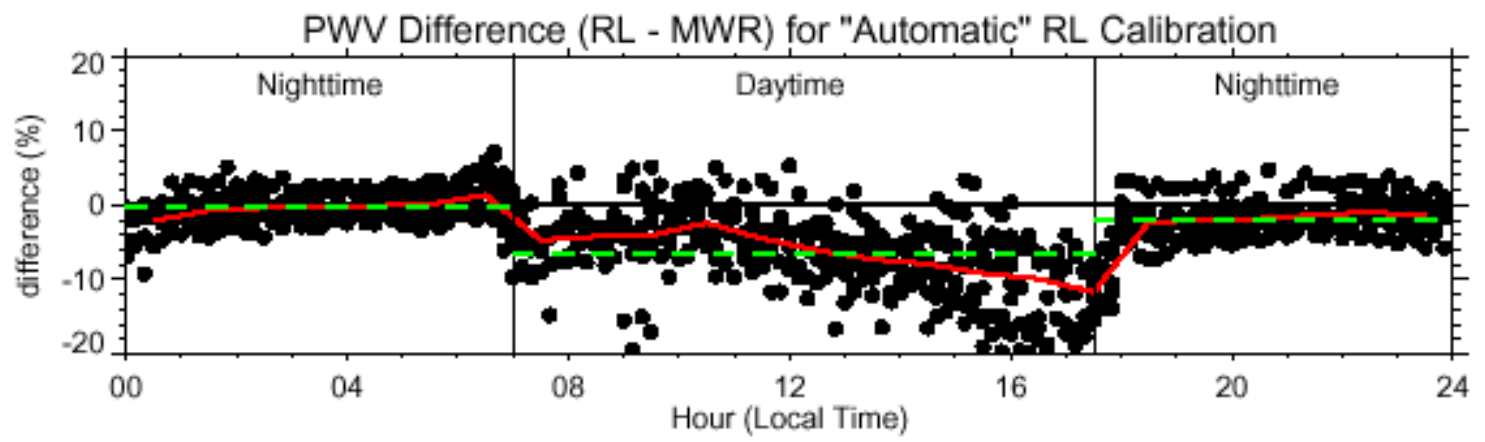

PWV Difference (RL - MWR) for "Manual" RL Calibration

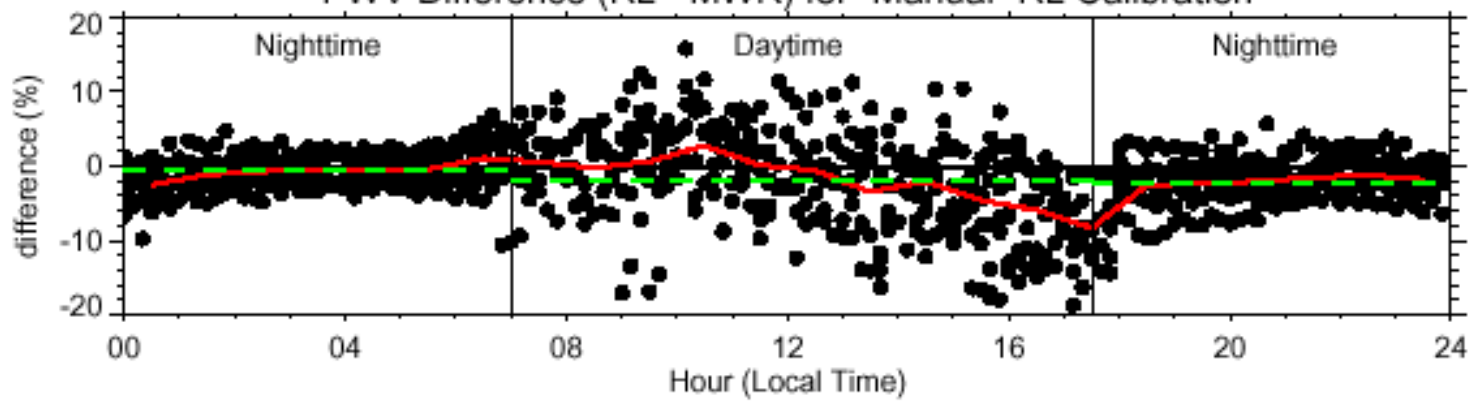

Figure 4. The normalized difference of the total PWV from the Raman lidar and MWR, for the original automated calibration results (top) and results where the relationship between the narrow and wide FOVs is manually determined (bottom). The solid red line indicates hourly averages of the differences, whereas the dashed green lines indicate mean values for each period. See text for details.

To isolate the cause of this, a more detailed analysis of the Raman lidar's data was completed. If the relationship between the narrow and wide FOVs is specified manually [using a value similar to that used in Turner and Goldsmith (1999)], rather than allowing the processing software to automatically determine it, the large diurnal difference in the Raman lidar data disappears (bottom panels of Figure 3 and Figure 4). However, the daytime differences with the MWR show an unexpected trend, with the differences changing more than $10 \%$ during the daytime hours, that is not understood at this time. 
By manually stating the relationship between the two FOVs, the mean diurnal differences between the Raman lidar - DIAL and the Raman lidar - MWR are reduced to less than $2 \%$. However, the daily mean difference between the Raman lidar and the DIAL is on the order of $10 \%$, while the daily mean Raman lidar and MWR difference is nearly zero. From these measurements, it cannot be concluded which is more accurate, the DIAL or the MWR-calibrated Raman lidar. However, the accuracy of the DIAL's measurements depends greatly on proper system performance. During this intensive operational period (IOP), the level of internal system checks were insufficient to claim that the DIAL's measurements were within a few percent accuracy, although there was no indication of system malfunction.

\section{Conclusion}

Detailed comparisons of the measurements from the two lidars indicate that both are very useful for detailed studies of atmospheric water vapor. Differences in performance are due to the different developmental status of both, as well as differences in methodology. Data from this IOP has helped identify and rectify a problem in the Raman lidar's processing scheme. It also has shown that there is a large discrepancy between two instruments that are capable of measuring water vapor with high absolute accuracy. An attempt to solve this problem will be made at the next intercomparison, which will occur at the CART site in 2000.

\section{Corresponding Author}

D. D. Turner, dave.turner@pnl.gov, Phone 509-375-2590, Fax 509-372-6247.

\section{References}

Clough, S. A., et al., 1999: Effect on the calculated spectral surface radiances due to MWR scaling of sonde water vapor profiles. In Proceedings of the Ninth Atmospheric Radiation Measurement (ARM) Science Team Meeting, U.S. Department of Energy, Washington, D.C. Available URL: http://www.arm.gov/docs/documents/technical/conf_9903/ clough-99.pdf

Goldsmith, J. E. M, F. H. Blair, S. E. Bisson, and D. D. Turner, 1998: Turn-key Raman lidar for profiling atmospheric water vapor, clouds, and aerosols. Appl. Opt., 37, 4979-4990.

Lehmann, S., V. Wulfmeyer, and J. Bösenberg, 1997: Time-dependent variable attenuator for dynamic range reduction in lidar signals. Appl. Opt., 36, 3469-3474.

Linné, H., D. D. Turner, J. E. M. Goldsmith, T. P. Tooman, J. Bösenberg, K. Ertel, and S. Lehmann, 2000: Intercomparison of DIAL and Raman lidar measurements of humidity profiles. In Proc. of the $20^{\text {th }}$ International Laser Radar Conference, Vichy, France.

Senff, C., J. Bösenberg, and G. Peters, 1994: Measurement of water vapor flux profiles in the convective boundary layer with lidar and radar-RASS. J. Atmos. Oceanic Technol, 11, 85-93. 
Sherlock, V., A. Hauchecorne, and J. Lenoble, 1999: Methodology for the independent calibration of Raman backscatter water-vapor lidar systems. Appl. Opt., 38, 5816-5837.

Turner, D. D., and J. E. M. Goldsmith, 1999: Twenty-four-hour Raman lidar water vapor measurements during the Atmospheric Radiation Measurement Program's 1996 and 1997 water vapor intensive observation periods. J. Atmos. Oceanic Technol., 16, 1062-1076.

Whiteman, D. N, T. Berkoff, D. D. Turner, T. P. Tooman, R. A. Ferrare, and L. A. Heilman, 2000: Research efforts in the absolute calibration of a Raman water vapor lidar. In Proc. of the $20^{\text {th }}$ International Laser Radar Conference, Vichy, France.

Wulfmeyer, V., and J. Bösenberg, 1998: Ground-based differential absorption lidar for water vapor profiling: assessment of accuracy, resolution, and meteorological applications. Appl. Opt., 37, 3825-3844. 